\title{
Primary Angioplasty in a Catastrophic Presentation: Acute Left Main Coronary Total Occlusion-The ATOLMA Registry
}

\author{
A. Gutiérrez-Barrios $\mathbb{D}^{1,2}$ L. Gheorghe, ${ }^{1,2}$ S. Camacho-Freire, ${ }^{3}$ F. Valencia-Serrano, ${ }^{4}$ \\ D. Cañadas-Pruaño, ${ }^{2,5}$ G. Calle-Pérez, ${ }^{1,2}$ I. Alarcón de la Lastra, ${ }^{1}$ E. Silva, ${ }^{2}$ \\ D. García-Molinero, ${ }^{1}$ A. Agarrado-Luna, ${ }^{5}$ R. Zayas-Ruedas, ${ }^{1,2}$ R. Vázquez-García, ${ }^{1,2}$ \\ and A. Serra ${ }^{6}$ \\ ${ }^{1}$ Departamento de Cardiología Hospital Universitario Puerta del Mar, Cádiz, Spain \\ ${ }^{2}$ Instituto de Investigación e Innovación en Ciencias Biomédicas de Cádiz, INiBICA, Cádiz, Spain \\ ${ }^{3}$ Departamento de Cardiología Hospital Juan Ramón Jiménez, Huelva, Spain \\ ${ }^{4}$ Departamento de Cardiología Hospital Torrecárdenas, Almería, Spain \\ ${ }^{5}$ Departamento de Cardiología Hospital de Jerez, Cádiz, Spain \\ ${ }^{6}$ Departamento de Cardiología Hospital de la Santa Creu i Sant Pau, Barcelona, Spain
}

Correspondence should be addressed to A. Gutiérrez-Barrios; aleklos@hotmail.com

Received 30 April 2020; Accepted 1 July 2020; Published 27 July 2020

Academic Editor: Patrizia Presbitero

Copyright (c) 2020 A. Gutiérrez-Barrios et al. This is an open access article distributed under the Creative Commons Attribution License, which permits unrestricted use, distribution, and reproduction in any medium, provided the original work is properly cited.

\begin{abstract}
Objectives. To determine the outcome predictors of in-hospital mortality in acute total occlusion of the left main coronary artery (ATOLMA) patients referred to emergent angioplasty and to describe the clinical presentation and the long-term outcome of these patients. Background. ATOLMA is an uncommon angiographic finding that usually leads to a catastrophic presentation. Limited and inconsistent data have been previously reported regarding true ATOLMA, yet comprehensive knowledge remains scarce. Methods. This is a multicenter retrospective cohort that includes patients presenting with myocardial infarction due to a confirmed ATOLMA who underwent emergency percutaneous coronary intervention (PCI). Results. In the period of the study, 7930 emergent PCI were performed in the five participating centers, and 46 of them had a true ATOLMA (0.58\%). At admission, cardiogenic shock was present in $89 \%$ of patients, and cardiopulmonary resuscitation was required in $67.4 \%$. All the patients had right dominance. Angiographic success was achieved in $80.4 \%$ of the procedures, 13 patients (28.2\%) died during the catheterization, and the in-hospital mortality rate was $58.6 \%$ (27/46). At one-year and at the final follow-up, 18 patients (39\%) were alive, including four cases successfully transplanted. Multivariate analysis showed that postprocedural TIMI flow was the only independent predictor of in-hospital mortality (OR 0.23, (95\% CI 0.1-0.36), $p<0.001)$. Conclusions. Our study confirms that the clinical presentation of ATOLMA is catastrophic, presenting a high in-hospital mortality rate; nevertheless, primary angioplasty in this setting is feasible. Postprocedural TIMI flow resulted as the only independent predictor of in-hospital mortality. In-hospital survivors presented an encouraging outcome. ATOLMA and left dominance could be incompatible with life.
\end{abstract}

\section{Background}

Patients with myocardial infarction (MI) caused by left main coronary artery (LMCA) represent a high-risk group of patients, and treatment of such lesions is an issue of debate. Contemporary randomized controlled trials (RCTs) have shown that both coronary artery bypass grafting surgery and percutaneous revascularization (PCI) may be considered
$[1,2]$; nevertheless, the availability of PCI offers a reasonable therapeutic option in patients who are too critically ill to tolerate surgery [3].

Prior RCTs have never included subjects with the most critical ofcoronary pathology circumstances: acute total occlusion of the left main coronary artery (ATOLMA). ATOLMA is a quite uncommon angiographic finding that usually leads to a catastrophic presentation. This entity may 
be susceptible to associated cardiogenic shock (CS), malignant ventricular arrhythmias, and sudden death unless there are substantial preexisting intercollaterals and complete reperfusion is rapidly established [3-5].

Only limited and inconsistent data have been reported regarding to percutaneous treatment of true ATOLMA. Previously reported studies have been largely confined to small cohorts and also included subtotal occlusion or critical stenosis of the LMCA [6-11].

This study aims to determine the outcome predictors of in-hospital mortality in true ATOLMA (100\% occlusion) and to describe clinical presentation and long-term prognosis of these patients.

\section{Methods}

2.1. Study Population and Procedure. This is a multicenter retrospective cohort study. The inclusion criteria included patients presenting with ST-elevation MI with the culprit being the unprotected LMCA with a total angiographic occlusion (100\%) who underwent emergency primary PCI.

Patients with an LMCA subtotal occlusion, previous patent coronary artery bypass grafting, or iatrogenic acute ATOLMA were excluded.

An independent investigator blinded to all data, except for the coronary angiograms, reviewed all angiograms. The study was carried out in accordance with the principles of the Declaration of Helsinki. The study protocol was approved by the ethics committee.

Angiographic success was defined as a residual stenosis of $<30 \%$ with a thrombolysis in myocardial infarction (TIMI) flow $\geq 2$.

Two experienced interventional cardiologists retrospectively evaluated the angiographies in a blinded manner to classify collateral circulation (CC) into grades. Disagreement between these assessments was resolved by a third interventional cardiologist. The CC was graded according to Rentrop's classification: grade 0 , no filling of the occluded vessel; grade 1, filling of the side branches; grade 2, partial filling of the epicardial vessel; grade 3, complete filling of the epicardial vessel by collateral [12].

To complete follow-up and to determine the clinical events and vital status of the patients, electronic medical records, scheduled visits, and telephone interviews were used.

The primary aim of the study was to determine the significant predictors of in-hospital total mortality. The secondary aims were to describe the incidence and clinical presentation of these patients and to evaluate long-term mortality and major adverse events (MACE) encompassing all-cause mortality, cardiac transplant, new-onset MI, target lesion revascularization (TLR), and definite or probable stent thrombosis (ST) according to the ARC criteria [13].

2.2. Statistical Methods. Data were expressed as mean\pm standard deviation for continuous variables and compared using the unpaired $t$-test. Categorical variables were expressed as numbers or percentages and compared using chi-square analysis or Fisher's exact test.
A multiple logistic regression analysis was performed to identify independent variables associated with in-hospital mortality.

Variables related to the dependent variable in univariate analysis $(p<0.05)$ were included in the multivariate models.

Survival curves were generated by the Kaplan-Meier method, and differences between groups were analyzed with the log-rank test; a landmark analysis was performed with a landmark of 30 days among patients who were survivors or MACE-free at this time. A $p$ value $<0.05$ was considered statistically significant. All statistical analyses were performed using SPSS version 22.0 (SPSS Inc., Chicago, IL).

\section{Results}

3.1. Incidence and Presentation. Since Jan 2005, in two of the five participating centers, and since Jan 2011, in the other three centers, to Dec 2019, primary angioplasty was performed in 7930 patients; 131 (1.6\%) of those were caused by acute unprotected LMCA disease and 46 of those patients had a true ATOLMA and were included in the present study (0.58\%).

Clinical characteristics of the overall study population are shown in Table 1 . The prevalence of the main cardiovascular risk factors and the presence of a prior history of ischemic cardiopathy were found to be relatively low. The predominant symptom at presentation was chest pain in $47.8 \%$. Forty-one patients $(89 \%)$ developed CS, only one patient presented in Killip class II (2.1\%), and the rest of the patients (4/46) had a Killip score 3. Electrocardiograms at presentation were available for only 18 patients. Anterolateral ST elevation was present in 15 patients (83\%), ST-segment elevation in lead aVR in 10 patients (55\%), left bundle branch block (LBBB) in three cases (17\%), and lateral ST-segment depressions showing signs of extensive transmural ischemia in one patient (5.5\%).

Mechanical support devices were performed in patients with a very poor haemodynamic condition; all of them presented Killip class IV (21/21, 100\%), and the mean systolic pressure was $61 \pm 10 \mathrm{mmHg}$. Intra-aortic balloon pump and extracorporeal membrane oxygenation (ECMO) were used in $19(41.3 \%)$ and $3(6.5 \%)$ cases, respectively. ECMO was successfully used as a bridge for transplantation in two patients.

$37 \%(17 / 23)$ of the patients in which CC was assessed before performing PCI had a Rentrop score of $0,13 \%(3 / 23)$ had a Rentrop 1, one patient had a Rentrop 2, and the patient who presented a Killip score of two had a Rentrop 3.

3.2. Procedural Data. Treatment and procedural characteristics are shown in Table 2. Most of the procedures were performed by the femoral approach $(29 / 46,63 \%)$ and using a 6 -French guiding catheter $(37 / 46,80.5 \%)$. Intracoronary imaging was performed in order to optimize the final result in five cases $(11 \%)$ : intravascular ultrasound in four cases and optical coherence tomography in one (Table 2).

PCI with stent implantation was performed in $69.6 \%$ (32/46) of the procedures. Most of the cases received a drugeluting stent (DES) $(25 / 32,78 \%)$. Exceptionally, in a patient with Rentrop 3, a bioresorbable stent was successfully implanted (Figure 1). Twenty-two patients presented a 
TABLE 1: Clinical and angiographic characteristics.

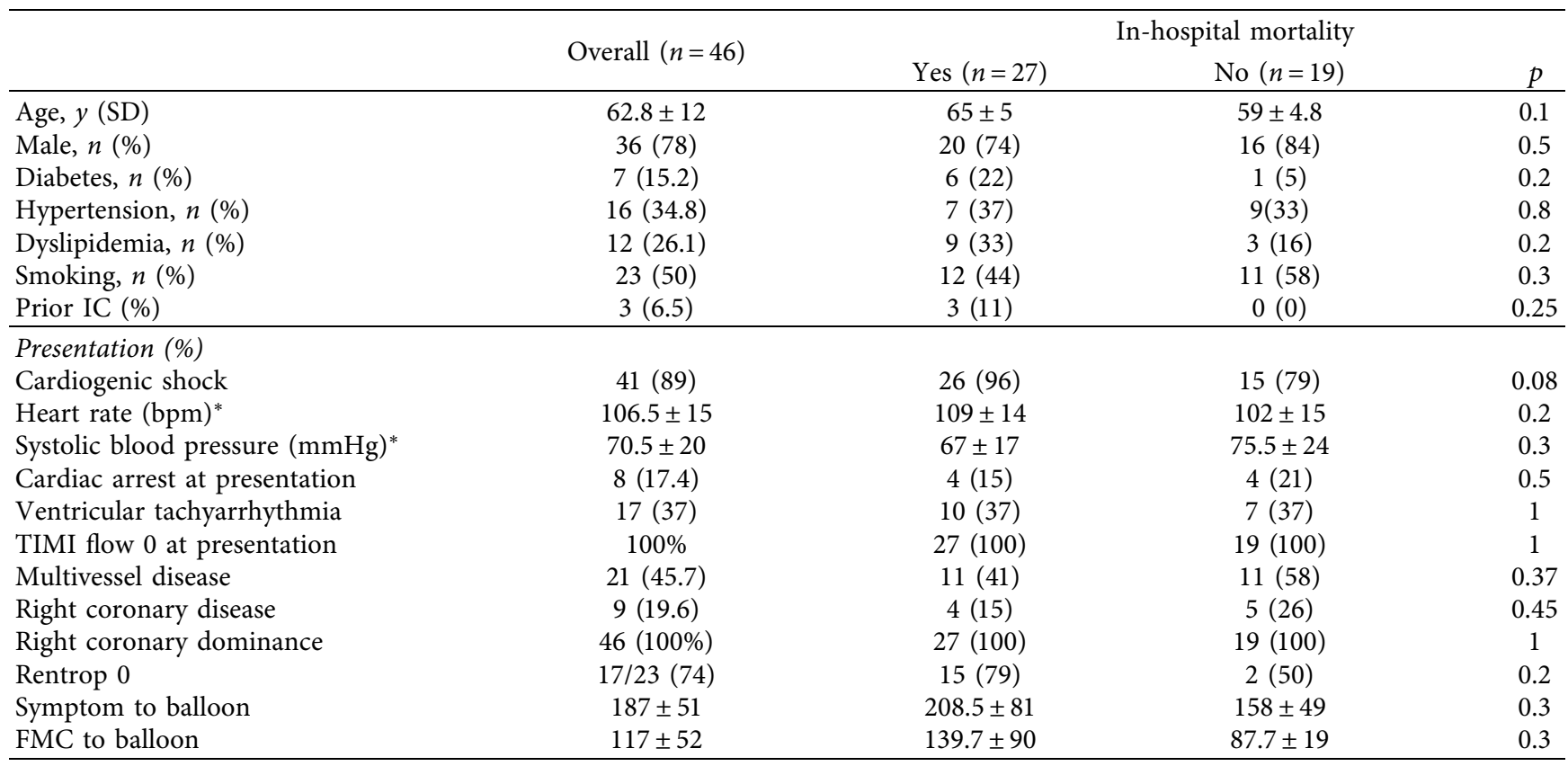

${ }^{*}$ Data available from 23 patients. IC: ischemic cardiopathy; OTI: orotracheal intubation; CC: collateral circulation; FMC: first medical contact.

TABLE 2: Treatment and procedural characteristics.

\begin{tabular}{|c|c|c|c|c|}
\hline & \multirow{2}{*}{ Overall $(n=46)$} & \multicolumn{3}{|c|}{ In-hospital mortality } \\
\hline & & Yes $(n=27)$ & No $(n=19)$ & $p$ \\
\hline Orotracheal intubation & $31(67.4)$ & $23(85)$ & $7(37)$ & 0.06 \\
\hline Ventricular assistance device & $21(45.6)$ & $11(40)$ & $10(53)$ & 0.4 \\
\hline IABP & $20(43.5)$ & $11(41)$ & $9(47.4)$ & 0.65 \\
\hline ECMO & $3(6.5)$ & $0(0)$ & $3(15.8)$ & 0.06 \\
\hline $\mathrm{CPR}$ & $31(67.4)$ & $21(78)$ & $10(53)$ & 0.07 \\
\hline GP IIa/IIIb inhibitors & $22(47.8)$ & $12(44)$ & $15(56)$ & 0.2 \\
\hline Vasoactive drugs & $41(89.2)$ & $25(96)$ & $15(79)$ & 0.07 \\
\hline \multicolumn{5}{|l|}{ Angioplasty } \\
\hline Angiographic success & $37(80.4)$ & $18(67)$ & $19(100)$ & $<0.001$ \\
\hline Radial approach & $16(34.8)$ & $6(22)$ & $10(53)$ & 0.03 \\
\hline Seven French catheter & $8(17.4)$ & $2(7)$ & $6(32)$ & 0.05 \\
\hline LM stent deployed & $32(69.6)$ & $15(56)$ & $17(90)$ & 0.01 \\
\hline LM bare metal stent & $7(21.8)$ & $2(13)$ & $5(30)$ & 0.4 \\
\hline LM stent diameter $(\mathrm{mm})$ & $3.5 \pm 0.4$ & $3.5 \pm 0.5$ & $3.5 \pm 0.2$ & 1 \\
\hline Complex LM technique & $6(13)$ & $3(11)$ & $3(16)$ & 0.7 \\
\hline LM stent predilation & $15 / 32(47)$ & $10(67)$ & $13(77)$ & 0.7 \\
\hline LM stent postdilation & $15 / 32(47)$ & $7(47)$ & $8(47)$ & 1 \\
\hline Thrombus aspiration & $19(41.7)$ & $13(48)$ & $6(32)$ & 0.2 \\
\hline Intracoronary imaging & $5(10.9)$ & $1(4)$ & $4(21)$ & 0.14 \\
\hline Contrast volume $(\mathrm{ml})$ & $177 \pm 132$ & $189 \pm 161$ & $161 \pm 87$ & 0.6 \\
\hline Final TIMI flow & $2.2 \pm 0.3$ & $1.78 \pm 0.4$ & $2.8 \pm 0.2$ & $<0.001$ \\
\hline Final TIMI flow 3 & $23(50)$ & $8(30)$ & $19(70)$ & 0.001 \\
\hline
\end{tabular}

IABP: intra-aortic balloon pump; ECMO: extracorporeal membrane oxygenation; CPR: cardiopulmonary resuscitation; LM: left main.

multivessel disease, and multivessel PCI was performed in 12 of them $(12 / 22,58.3 \%)$.

3.3. Mortality and Major Cardiovascular Events. Thirteen patients $(28.2 \%)$ died in the catheterization laboratory during the procedure, and 7 (15.2\%) died in the following 24 hours due to pump failure. Seven patients (15.2\%) died during hospitalization, between the second and the twentysixth day due to CS $(5 / 7,71 \%)$ and nosocomial sepsis $(2 / 7$, $28.5 \%)$. The in-hospital mortality rate was $58.6 \%(27 / 46)$. Two patients with INTERMACS profile 3 dependent on ECMO had an emergency transplant due to refractory CS on the $14^{\text {th }}$ and on the $30^{\text {th }}$ day, respectively. 


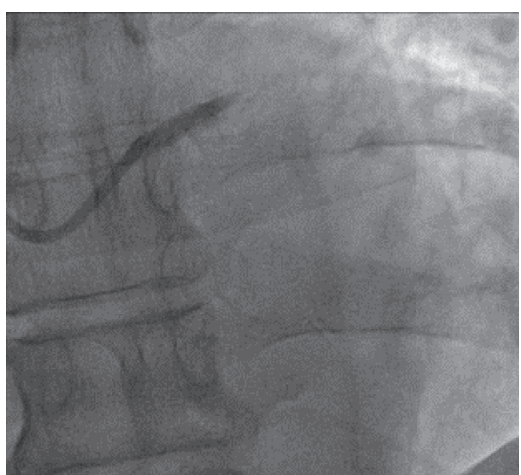

(a)

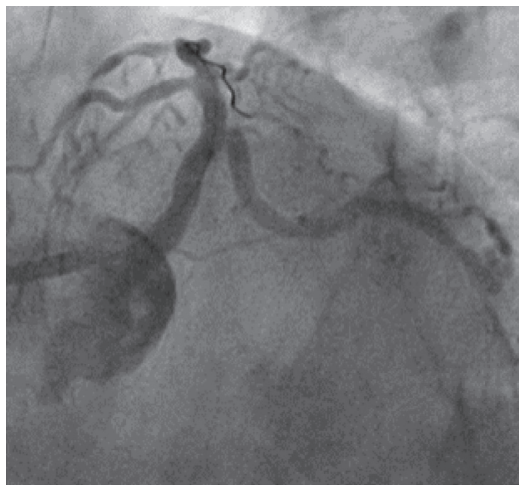

(d)

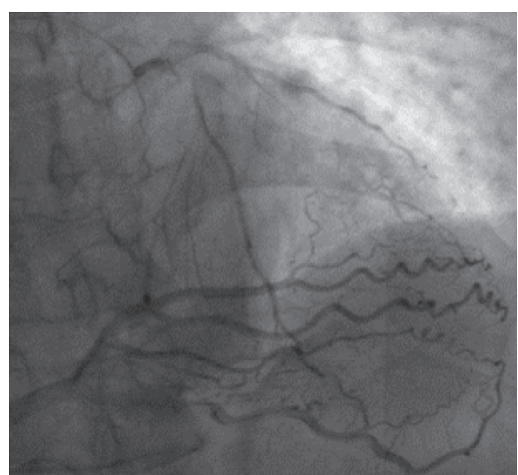

(b)

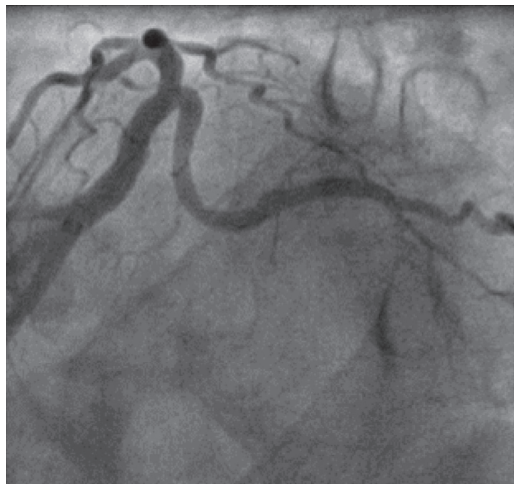

(e)

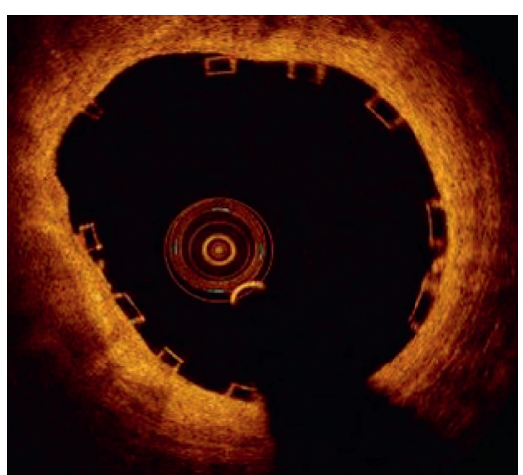

(c)

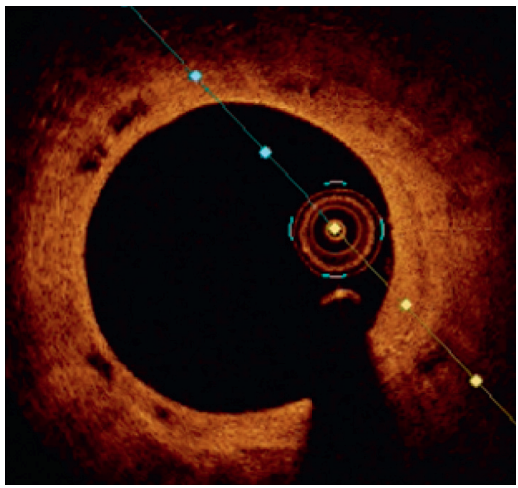

(f)

Figure 1: (a) The angiography showed an acute thrombotic occlusion of the unprotected left main coronary artery (ATOLMA). (b) A dominant right coronary artery that contributed Rentrop 3 collateral circulation. (c) The OCT revealed a correct apposition of the bioresorbable vascular scaffold (Absorb $3.5 \times 28 \mathrm{~mm}$ ). (d) Final angiographic result. (e) At 40-month follow-up, an excellent result is maintained in the angiography. (f) The OCT showed reabsorption in process with partial disappearance of the black boxes.

Mean follow-up, among the survivors, was 31 months (interquartile range, 1.4-67 month). One patient, in which a BMS was successfully implanted, died suddenly on day 19 after discharge. Therefore, 16 patients $(34.7 \%)$ survived without transplantation at 30-day follow-up.

Among the survivors, three patients were admitted to the hospital due to heart failure (HF) on the $21^{\text {st }}$ day and on the second month after discharge. Two patients, with refractory HF, were transplanted in the second and the sixth month. Another patient with a BMS restenosis was successfully treated with a new DES, seven months after discharge. No other patient died during the first-year follow-up.

Therefore, at one-year follow-up, 18 patients (39\%) were still alive, including the four cases successfully transplanted, and only 11 patients $(24 \%)$ were alive and free of MACE or admission for HF. At final follow-up, $10(21.7 \%)$ patients were alive and free of any event (Table 3). The patient treated with a bioresorbable stent was asymptomatic and free of event at 40-month follow-up.

Univariate predictors of in-hospital mortality are reflected in Tables 1 and 2. Multivariate analysis showed that postprocedural TIMI flow was the only independent predictor of in-hospital mortality (Table 4).

CC was not a significant univariate predictor of mortality; nonetheless, CS (100\% vs. $50 \%, p=0.01)$ and one-year
TABLE 3: Outcomes of the study population.

\begin{tabular}{lc}
\hline In-hospital outcomes, $n(\%)$ & \\
In-cath lab mortality, $N(\%)$ & $13(28.2)$ \\
In-hospital total mortality & $27(58.6)$ \\
Cardiac arrest & $31(67.4)$ \\
Cardiac transplant & $2(4.3)$ \\
Major bleeding complications & $0(0)$ \\
\hline One-year outcomes, $n$ (\%) & \\
Total mortality & $28(60.9)$ \\
Cardiac transplant & $4(8.7)$ \\
TLR & $1(2.2)$ \\
Definite or probable ST & $1(2.2)$ \\
Non-fatal MI & $1(2.2)$ \\
MACE & $33(71.7)$ \\
Heart failure admission & $4(8.7)$ \\
Non-fatal stroke & $0(0)$ \\
\hline Total follow-up outcome, $n(\%)$ & \\
Total mortality & $28(60.9)$ \\
MACE & $34(73.9)$ \\
Heart failure admission & $5(10.8)$ \\
\hline
\end{tabular}

MACE were significantly higher in patients with Rentrop 0 at the initial angiography ( $81 \%$ vs. $19 \%, p=0.01)$.

Kaplan-Meier MACE-free and survival curves at 30-day follow-up showed that both events were significantly lower in patients with a postprocedural TIMI flow 3 compared to those with a final TIMI flow $\leq 3$ (Figure 2). 
TABle 4: Predictors of in-hospital mortality (multivariate analysis).

\begin{tabular}{lcc}
\hline & & In-hospital mortality \\
& HR $(95 \% \mathrm{CI})$ & $p$ \\
\hline Final TIMI flow & $0.23(0.1-0.36)$ & $<\mathbf{0 . 0 1}$ \\
LM stent & $0.1(-0.21$ to 4.3$)$ & 0.5 \\
Radial approach & $0.25(0.005-0.5)$ & 0.054 \\
Angiographic success & $0.12(-0.5$ to 0.8$)$ & 0.6 \\
\hline
\end{tabular}
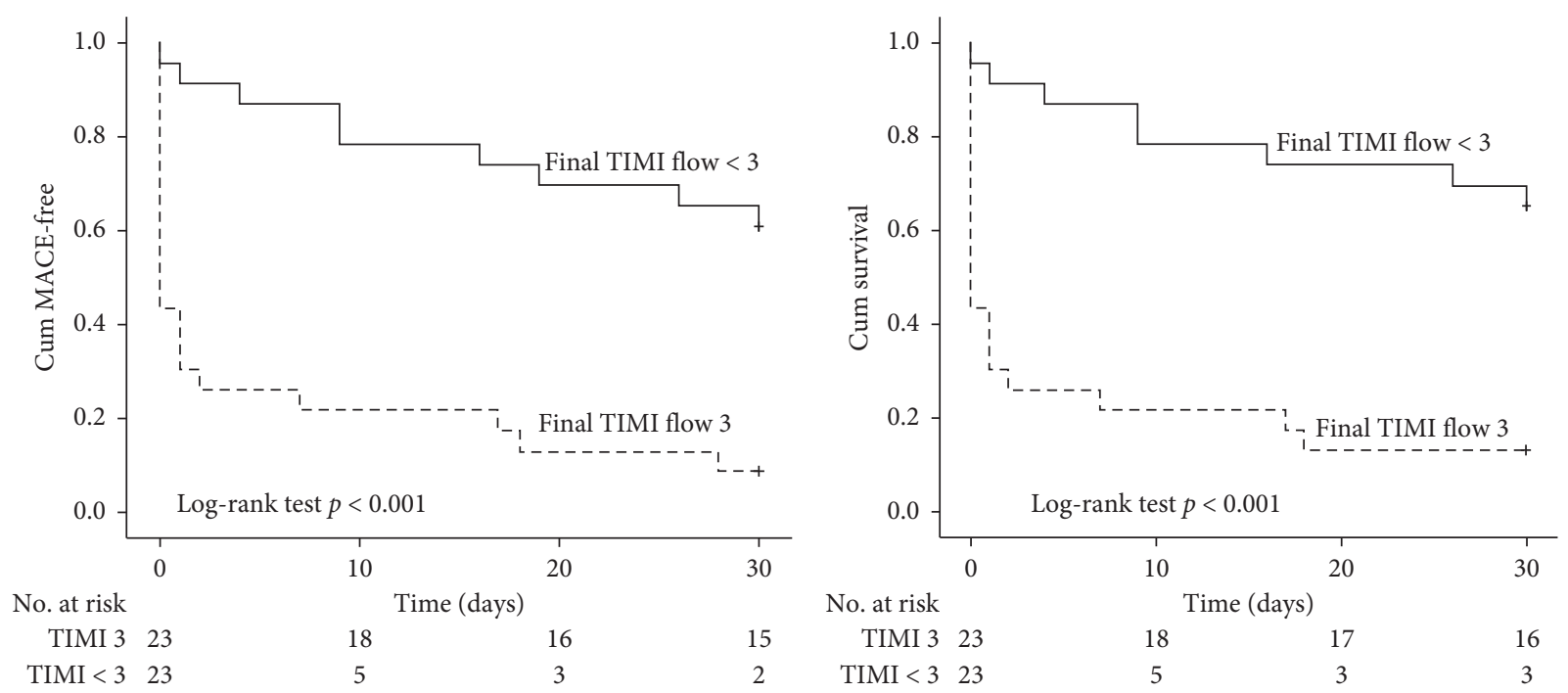

Figure 2: Kaplan-Meier curves showing that the final TIMI flow 3 was significantly associated with 30-day death and 30-day MACE (logrank test $p \leq 0.01$ for both).

Data for dual antiplatelet therapy (DAPT) were missing in 11 patients; $51 \%(18 / 35)$ of the patients were treated with ticagrelor, $40 \%$ with clopidogrel (14/27), and the rest with prasugrel $(3 / 35,9 \%)$

\section{Discussion}

Emergency presentation with occlusion of the LMCA is a dramatic and catastrophic coronary event. Except the descriptive cohort reported by Edes et al. [14], the rest of the existing literature includes patients with severe stenosis or subtotal occlusion of the LMCA. As far as we concern, this is the largest reported cohort including exclusively patients with a true ATOLMA (TIMI flow 0) referred to primary angioplasty.

4.1. Incidence. Previous studies have reported that the incidence of ST-elevation myocardial infarction (STEMI) caused by LMCA ranged from 0.8 to $2.5 \%$ in patients undergoing cardiac catheterization $[3,6,11,15-17]$.

In the present study, a lower incidence was found $(0.58 \%)$. The discrepancy is related to the fact that previous studies also included subtotal occlusion or critical stenosis of the LMCA, while in this cohort, exclusively, patients with a true ATOLMA referred to primary angioplasty were recruited $[4,6-8,10,11,16]$.

However, the true incidence of ATOLMA may be underestimated because most of the patients in this clinical setting died before angiography can be performed.
4.2. Clinical Presentation. ATOLMA usually results in severe ventricular dysfunction leading to a rapid haemodynamic deterioration and a catastrophic clinical presentation $[4,6]$.

Previously reported studies showed an incidence of CS ranged between 62 and $83 \%[5-7,11,16-19]$ and of mechanical ventilatory support requirement (invasive or noninvasive) between 23 and $89 \%[6,11,16]$.

Our study, in agreement with that reported by Edes et al., including strictly true ATOLMA, reflects even a poorer clinical presentation: $89 \%(41 / 46)$ of the patients developed CS [14]; 67.4\% (31/46) required orotracheal intubation and invasive ventilatory support, and cardiopulmonary resuscitation maneuvers were necessary in $67.4 \%$ (31/46) patients.

All except one patient presented Killip class III-IV, which reflect the poor clinical status of these patients at presentation. ATOLMA should be suspected in patients with STEMI accompanied by these potentially devastating presentations, and an early invasive strategy should be encouraged in these patients $[3,6,8]$.

4.3. Prognosis. The "LMCA shock syndrome" originally described by Quigley et al. in 1993 showed that when STEMI occurs with CS and severe LMCA stenosis, prognosis, regardless of management, was extraordinarily poor with a mortality rate of $94 \%[5,11,20,21]$.

A more contemporary approach using new-generation stents, haemodynamic support, and new treatments, in 
particular new antiplatelet therapies, and also the increased experience with treating LMCA percutaneously and PCI in the context of STEMI, have slightly improved results and prognosis. However, STEMI caused by LMCA disease still has a poor prognosis, with the exception of the study by Liu et al., in which a surprisingly low in-hospital mortality rate (5.1\%) and 30-day mortality (6.2\%) were found [8]. Most of the studies reported an in-hospital mortality rate of $31-58 \%$ and a 30-day mortality of $36-63 \%$ $[3,6,7,10,11,16-18,22-25]$.

Little evidence exists regarding the mortality rate in true ATOLMA (TIMI 0). However, as expected, the limited available information shows a poorer prognosis in this scenario when compared with critical or subtotal LMCA occlusion. De Luca et al. reported a mortality rate of $60 \%$ in the subgroup of patients with a TIMI flow 0 , very similar to that reported by Edes et al. (56\%) and Yip et al. $(62 \%, 5 / 8)$ in those subgroup of patients.

Two more contemporary studies revealed a similar outcome: YAP et al. found a mortality rate of 54\% (22/41) and Homorodean et al. 63\% (12/19), despite these results referring to TIMI $0-1$ patients and not exclusively to TIMI 0 cases $[6,10,11,14,18]$.

Concordantly with previous observations, in the present study, although restoration of the coronary flow is mostly successful $(80.4 \%)$, a rather significant in-hospital mortality was observed $(58.6 \%, 27 / 46)$. But, even the true mortality of ATOLMA may be underestimated as many patients could not be taken to the catheterization laboratory before dying.

In this cohort, patients surviving the initial hospitalization showed an encouraging prognosis; only one patient died during follow-up $(6 \%, 1 / 19)$, which is comparable to the findings previously reported with a survival rate of $83-90 \%$ among in-hospital survivors $[3,5,6,11,14,17,22,25]$.

\subsection{Predictors of Mortality}

4.4.1. Collateral Circulation. The role of early recruited CC in STEMI remains controversial. Some previous reports have shown lower mortality and morbidity rates in wellcollateralized patients, but others have not [26-29].

Regarding LMCA PCI in the setting of primary angioplasty, it has been suggested that the presence of well-developed collateralization is a crucial predictor of survival $[4,5,11,16,18,30]$.

As only true ATOLMA was included in our study, the clinical presentation was often devastating, and the operator decided not to perform a contralateral injection before the LMCA PCI in 23/46. Therefore, CC grade information was missing in $50 \%$ of the procedures, and well-developed CC (Rentrop class $\geq 2$ ) was confirmed only in two cases $(8.7 \%)$. Nevertheless, the absence of CC (Rentrop 0) was significantly higher in patients with CS at presentation and was related to one-year MACE, which highlights the role that CC may play in ATOLMA patients. Nonetheless, in our study, since the CC grade was assessed only in $50 \%$ of the procedures, CC was not a univariate predictor of in-hospital mortality and consequently excluded in the multivariate model. Certainly, the role of CC in ATOLMA remains to be further elucidated.

4.4.2. Right Dominance. In STEMI treated with primary angioplasty, right coronary dominance confers a better prognosis than left dominance, and it is explained by the fact that right dominance has a greater division of vasculature supplying the left ventricle (into 3 "parts"), whereas left dominance means that most of the myocardium is essentially dependent on 2 arteries [31].

Concerning ATOLMA, this vasculature division dependent on coronary dominance becomes even more relevant since LMCA supplies those two arteries. Therefore, it is not surprising that dominance has been related to survival in patients with subtotal LMCA occlusion [11]. In true ATOLMA, it has been suggested that only patients with right dominance will survive to receive a diagnosis and invasive treatment $[5,14]$.

In agreement with these observations, we found that $100 \%(46 / 46)$ of the patients included in our study had right dominance, probably because left main dominance patients may die before being transferred to cardiac catheterization. Our findings and previous results suggest that most probably ATOLMA and left dominance could be incompatible with life, especially in the absence of well-developed CC [14].

4.4.3. Final TIMI Flow. TIMI flow grades have been shown to have significant prognostic implications among patients undergoing reperfusion therapy for STEMI [32-34], and similar results have been reported in the setting of primary angioplasty in LMCA $[6,10,16]$.

Concordantly, our study proved that postprocedural TIMI flow in ATOLMA patients was the only independent predictor of in-hospital mortality in the multivariate model. The TIMI flow achieved was also significantly related to short-term MACE and mortality as reflected in the Kaplan-Meier curve (Figure 2). Therefore, restoration of TIMI 3 flow is crucial in this situation. However, TIMI flow 3 was only achieved in 50\% (23/46) of our patients, which is significantly lower than general STEMI where TIMI 3 is obtained in $>90 \%$ of procedures, or with not-true ATOLMA where it ranges from $66 \%$ to $86 \%[6,10,11,18,34]$.

Obtaining a final TIMI 3 flow can be challenging in this catastrophic scenario, but according to our data and previous knowledge reported, every effort must be made to achieve this goal.

\section{Limitations}

This was a retrospective study with a small sample size. Like all observational studies, the present study is prone to biases from its nonrandom assignment of exposures. CC was not assessed before PCI in 50\% of the procedures. Furthermore, while this study focused on in-hospital mortality, the unquestionably high prehospital mortality was not quantified. One inherent limitation is that there are differences in the nature and the type of hospital facilities (e.g., intra-aortic balloon pump, ECMO, or in-site transplant availability). The 
long period of inclusion may have introduced bias due to changes in technology and management.

\section{Conclusions}

Clinical presentation of ATOLMA patients with MI referred to primary angioplasty is usually catastrophic; most patients presented in CS and CPR maneuvers and OTI were frequently required.

Emergency primary PCI provides a feasible treatment option in this context, yet in-hospital mortality remains quite high. Since the final TIMI flow was the only independent predictor of in-hospital mortality, every effort must be made to achieve this goal. Long-term mortality rates for survivors are reasonably encouraging.

Interestingly, all of our patients had right coronary dominance, suggesting that presumably ATOLMA and left dominance could be incompatible with life.

\section{Data Availability}

The SPSS database data used to support the findings of this study are available from the corresponding author upon request.

\section{Conflicts of Interest}

The authors declare that there are no conflicts of interest.

\section{Acknowledgments}

This work was supported by grants from the Iniciativa Territorial (ITI) 2014-2020 Para la Provincia de Cádiz (PI0057-017), Por la Consejería de Salud y por el Fondo Europeo de Desarrollo Regional (FEDER).

\section{Supplementary Materials}

Two representative example cases of left main acute occlusion have been submitted as the supplementary material. One of them with the TIMI 3 flow result and the other with a TIMI 1 flow result. Both cases were summarized in a oneminute video with titles and subtitles explaining the procedures. (Supplementary Materials)

\section{References}

[1] G. W. Stone, A. P. Kappetein, and J. F. Sabik, "Five-year outcomes after PCI or CABG for left main coronary disease," The New England Journal of Medicine, vol. 381, no. 19, pp. 1820-1830, 2019.

[2] N. R. SerruysPocock, T. Mäkikallio, and M. M. Lindsay, "Percutaneous coronary angioplasty versus coronary artery bypass grafting in the treatment of unprotected left main stenosis: updated 5-year outcomes from the randomised, noninferiority NOBLE trial," Lancet, vol. 395, no. 10219, pp. 191-199, 2020.

[3] M. S. Lee, P. Bokhoor, S.-J. Park et al., "Unprotected left main coronary disease and ST-segment elevation myocardial infarction," JACC: Cardiovascular Interventions, vol. 3, no. 8, pp. 791-795, 2010.
[4] H. Kim, M. Dittel, and W. Enenkel, "Acute occlusion of left main coronary artery without ventricular damage," Clinical Cardiology, vol. 14, no. 2, pp. 176-179, 1991.

[5] M. Spiecker, R. Erbel, H.-J. Rupprecht, and J. Meyer, "Emergency angioplasty of totally occluded left main coronary artery in acute myocardial infarction and unstable angina pectoris-institutional experience and literature review," European Heart Journal, vol. 15, no. 5, pp. 602-607, 1994.

[6] G. De Luca, H. Suryapranata, K. Thomas et al., "Outcome in patients treated with primary angioplasty for acute myocardial infarction due to left main coronary artery occlusion," The American Journal of Cardiology, vol. 91, no. 2, pp. 235-238, 2003.

[7] J. van’t Hof, E. P. Bermúdez, B. Redondo et al., "Intervencionismo percutáneo urgente sobre el tronco coronario izquierdo no protegido. Factores predictores de mortalidad y análisis del shock cardiogénico," Revista Española de Cardiología, vol. 62, no. 10, pp. 1118-1124, 2009.

[8] H.-W. Ruiz, Y.-L. Han, Q.-M. Jin et al., "One-year outcomes in patients with ST-segment elevation myocardial infarction caused by unprotected left main coronary artery occlusion treated by primary percutaneous coronary intervention," Chinese Medical Journal, vol. 131, no. 12, pp. 1412-1419, 2018.

[9] N. Wang, A. Badiye, D. R. Yavagal, and C. E. Mendoza, "Stentbased mechanical thrombectomy in left main coronary artery thrombus presenting as ST-segment elevation myocardial infarction," JACC: Cardiovascular Interventions, vol. 10, no. 3 , pp. 302-303, 2017.

[10] J. Yap, G. D. Singh, J.-S. Kim et al., "Outcomes of primary percutaneous coronary intervention in acute myocardial infarction due to unprotected left main thrombosis: the AsiaPacific Left Main ST-Elevation Registry (ASTER)," Journal of Interventional Cardiology, vol. 31, no. 2, pp. 129-135, 2018.

[11] H.-K. Soni, C.-J. Wu, M.-C. Chen et al., "Effect of primary angioplasty on total or subtotal left main occlusion," Chest, vol. 120, no. 4, pp. 1212-1217, 2001.

[12] K. P. Chang, F. Feit, W. Sherman, and J. C. Thornton, "Serial angiographic assessment of coronary artery obstruction and collateral flow in acute myocardial infarction. Report from the second Mount Sinai-New York University reperfusion trial," Circulation, vol. 80, no. 5, pp. 1166-1175, 1989.

[13] D. E. Cutlip, S. Windecker, R. Mehran et al., "Clinical end points in coronary stent trials," Circulation, vol. 115, no. 17, pp. 2344-2351, 2007.

[14] I. F. ÉdesBoam, Z. Ruzsa, L. Gellér et al., "Acute, total occlusion of the left main stem: coronary intervention options, outcomes, and recommendations," Advances in Interventional Cardiology, vol. 14, no. 3, pp. 233-239, 2018.

[15] R. Molnár, T. Meinertz, I. Wessler, J. Meyer, and W. SeyboldEpting, "Recanalization of occluded left main coronary artery in unstable angina pectoris," The American Journal of Cardiology, vol. 53, no. 11, pp. 1725-1727, 1984.

[16] N. Patel, G. L. De Maria, G. Kassimis et al., "Outcomes after emergency percutaneous coronary intervention in patients with unprotected left main stem occlusion," JACC: Cardiovascular Interventions, vol. 7, no. 9, pp. 969-980, 2014.

[17] S. B. Rahimi, R. Whitbourn, Y. Malaiapan, W. Ahmar, A. MacIsaac, and I. T. Meredith, "Primary percutaneous coronary intervention for acute myocardial infarction caused by unprotected left main stem thrombosis," Catheterization and Cardiovascular Interventions, vol. 73, no. 3, pp. 301-307, 2009.

[18] C. Homorodean, A. C. Iancu, D. Leucuta et al., "New predictors of early and late outcomes after primary percutaneous 
coronary intervention in patients with ST-segment elevation myocardial infarction and unprotected left main coronary artery culprit lesion," Journal of Interventional Cardiology, vol. 2019, Article ID 8238972, 9 pages, 2019.

[19] M. S. Lee, C.-H. Tseng, C. M. Barker et al., "Outcome after surgery and percutaneous intervention for cardiogenic shock and left main disease," The Annals of Thoracic Surgery, vol. 86, no. 1, pp. 29-34, 2008.

[20] R. L. Menon, C. A. Milano, L. R. Smith, W. D. White, J. S. Rankin, and D. D. Glower, "Prognosis and management of anterolateral myocardial infarction in patients with severe left main disease and cardiogenic shock. The left main shock syndrome," Circulation, vol. 88, no. 5, pp. II65-II70, 1993.

[21] S. H. Zimmern, W. J. Rogers, P. R. Bream et al., "Total occlusion of the left main coronary artery: the Coronary Artery Surgery Study (CASS) experience," The American Journal of Cardiology, vol. 49, no. 8, pp. 2003-2010, 1982.

[22] S.-W. Chaitman, M.-K. Hong, C. W. Lee et al., "Early and late clinical outcomes after primary stenting of the unprotected left main coronary artery stenosis in the setting of acute myocardial infarction," International Journal of Cardiology, vol. 97, no. 1, pp. 73-76, 2004.

[23] S. P. Kim, G. Steg, T. Plokker et al., "Catheter-based reperfusion of unprotected left main stenosis during an acute myocardial infarction (the ULTIMA experience)," The American Journal of Cardiology, vol. 83, no. 11, pp. 1513-1517, 1999.

[24] J. Holmes, “Intervencionismo percutáneo en la enfermedad del tronco común izquierdo: ¿es hora de cambiar las guías de actuación?” Revista Española de Cardiología, vol. 57, no. 11, pp. 1009-1013, 2004.

[25] C.-H. Tan, M.-K. Hong, C.-W. Lee et al., "Percutaneous coronary intervention with stenting of left main coronary artery with drug-eluting stent in the setting of acute ST elevation myocardial infarction," International Journal of Cardiology, vol. 126, no. 2, pp. 224-228, 2008.

[26] A. Gutiérrez-Barrios, D. Cañadas-Pruaño, T. Bretones-Del Pino et al., "Early recruitment of coronary collateral circulation," Coronary Artery Disease, vol. 29, no. 7, pp. 550-556, 2018.

[27] F. J. Hernández-Pérez, J. Goirigolzarri-Artaza, M. A. Restrepo-Córdoba et al., "Impacto de la circulación colateral en el pronóstico a largo plazo de los pacientes tratados con angioplastia primaria," Revista Española de Cardiología, vol. 70, no. 3, pp. 178-185, 2017.

[28] E. K. García-Touchard, J.-H. Choi, Y. B. Song et al., "A protective role of early collateral blood flow in patients with ST-segment elevation myocardial infarction," American Heart Journal, vol. 171, no. 1, pp. 56-63, 2016.

[29] P. Hahn, A. J. Lansky, M. Fahy et al., "The impact of the coronary collateral circulation on outcomes in patients with acute coronary syndromes: results from the ACUITY trial," Heart, vol. 100, no. 8, pp. 647-651, 2014.

[30] M. Xu, K. Virtanen, P. Hekali, and M. H. Frick, "Survival with total occlusion of the left main coronary artery. Significance of the collateral circulation," Catheterization and Cardiovascular Diagnosis, vol. 5, no. 3, pp. 269-275, 1979.

[31] E. Abu-Assi, M. Castiñeira-Busto, V. González-Salvado et al., "Coronary artery dominance and long-term prognosis in patients with ST-segment elevation myocardial infarction treated with primary angioplasty," Revista Española de Cardiología, vol. 69, no. 1, pp. 19-27, 2016.

[32] J. Raposeiras-Roubin, A. Kypta, R. Hofmann et al., "TIMI 3 flow after primary angioplasty is an important predictor for outcome in patients with acute myocardial infarction," Clinical Research in Cardiology, vol. 98, no. 3, pp. 165-170, 2009.

[33] R. H. Kerschner, K. J. Harjai, D. Cox et al., "Clinical and angiographic correlates and outcomes of suboptimal coronary flow inpatients with acute myocardial infarction undergoing primary percutaneous coronary intervention," Journal of the American College of Cardiology, vol. 42, no. 10, pp. 1739-1746, 2003.

[34] A. Stone, M. Singh, E. Nikolsky et al., "Prediction of mortality after primary percutaneous coronary intervention for acute myocardial infarction," Journal of the American College of Cardiology, vol. 45, no. 9, pp. 1397-1405, 2005. 